\section{BIBLIOGRAFÍA}

1. Rashid RM, Khachemoune A. Trigeminal trophic syndrome. J Eur Acad Dermatol Venereol 2007;21:725-731.

2. Fernández-Bernáldez A, Rodríguez-Jiménez $P$, HerreroMoyano M, Sánchez-Pérez J. Síndrome trófico trigeminal secundario a recidiva de meningioma. Actas Dermosifiliogr 2020;111:171-173.

3. Garza I. The trigeminal trophic syndrome: an unusual cause of face pain, dysaesthesias, anesthesia and skin/ soft tissue lesions. Cephalalgia 2008;28:980-985.

4. Khan AU, Khachemoune A. Trigeminal trophic syndrome: an updated review. Int J Dermatol 2019;58:530-537.

5. Sawada T, Asai J, Nomiyama T, Masuda K, et ál. Trigeminal trophic syndrome: Report of a case and review of the published work. J Dermatol 2014;41:525-528.

6. Luna P, Nocito M, Cañadas N, Castellanos Posse ML, et ál. Síndrome trófico trigeminal. Una causa infrecuente de úlceras faciales. Arch Argent Dermatol 2009;59:21-23.

7. Sadeghi P, Papay FA, Vidimos AT. Trigeminal trophic syndrome - report of four cases and review of the literature. Dermatol Surg 2004;30:807-812.

8. Khan FA, Manacheril R, Ulep R, Martin JE, et ál. Trigeminal trophic syndrome associated with the use of synthetic marijuana. Ochsner J 2017;17:292-295.

9. Gómez de Castro C, Vázquez-López F, García-García B, Requena López $\mathrm{S}$, et ál. Trigeminal trophic syndrome simulating rodent ulcer basal cell carcinoma: a new clinico-dermoscopic approach. An Bras Dermatol 2017;92:148-150.

10. Oaklander AL. Common neuropathic itch syndromes. Acta Derm Venereol 2012;92:118-125.

11. Zambernardi A, Digilio M, Spiner $R$, Feinsilber D, et ál. Síndrome trófico del trigémino. Un caso con extensión infrecuente. Dermatol Argent 2018;24:57-59.

12. Sallabanda K, Sallabanda M, Barrientos HD, Santaolalla $\mathrm{I}$, et ál. Trigeminal trophic syndrome secondary to refractory trigeminal neuralgia treated with CyberKnife ${ }^{\circledR}$ radiosurgery. Cureus 2020;12:e7670.

\title{
DERMATÓLOGOS JÓVENES
}

\section{* Elección múltiple: PLACA ERITEMATOVIOLÁCEA EN UN DEDO}

Agostina Alonzo Caldarelli, Pilar Targize Vaquero y Marlene Goland Servicio de Dermatología, HIGA Prof. Dr. Rodolfo Rossi, La Plata, Provincia de Buenos Aires, Argentina

Una mujer de 37 años, sin antecedentes patológicos de relevancia, consultó por la presencia de una lesión en el pulpejo del segundo dedo izquierdo de 8 meses de evolución, asintomática, de comienzo abrupto y tamaño estable, no relacionada con un traumatismo previo, sangrante ante el roce mínimo.

En el examen físico se observaba una placa de color rojo violáceo, de $7 \mathrm{~mm}$ de diámetro mayor, con bordes bien definidos, superficie irregulary una pústula central. No había frémito ni aumento de la temperatura local (Foto 1).

La dermatoscopia reveló un patrón monomorfo compuesto por múltiples lagunas rojo-lechosas, bien delimitadas, de diferentes tamaños, distribuidas en toda la lesión (Foto 2).

\footnotetext{
1) ¿Cuál es su diagnóstico más probable?

a) Hemangioendotelioma retiforme.

b) Angioma.

c) Angioqueratoma solitario.

d) Malformación arteriovenosa digital acral.

e) Hemangioma capilar lobulillar.
}

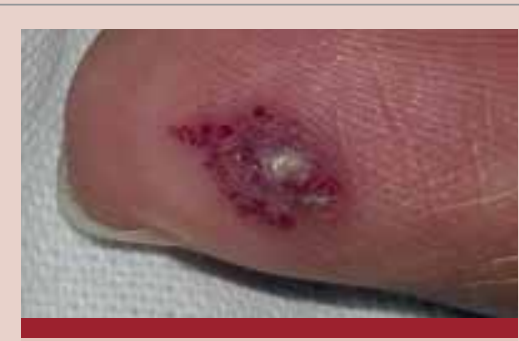

FOTO 1.

2) ¿Qué estudio solicitaría para arribar al diagnóstico definitivo?

a) Ecografía Doppler arterial y venosa.

b) Estudio histopatológico.

c) Radiografía de la mano.

d) Laboratorio con recuento de plaquetas.

e) Las opciones c. y d. son correctas.

3) ¿Qué esperaría encontrar en el estudio histopatológico?

a) Conductos vasculares arborizantes con patrón retiformey células endoteliales en forma de tachuela.

b) Vasos dérmicos entrelazados que forman un ovillo capilar.

c) Vasos subepidérmicos dilatados asociados con acantosis e hiperqueratosis.

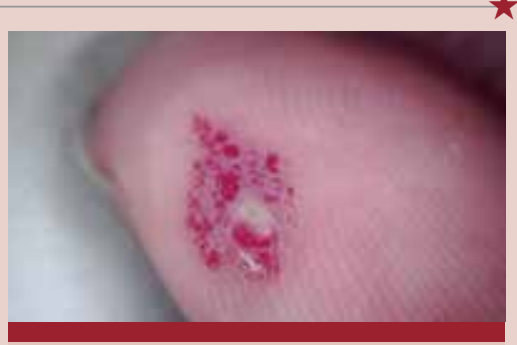

FОTO 2.

d) Múltiples interconexiones entre las arterias de mediano o gran calibre y las venas, sin interposición del lecho capilar.

e) Lobulillos pequeños de capilares dispuestos en una matriz fibromixoide, exofítica y delimitados por un collarete epidérmico hiperplásico.

4) ¿Qué tratamiento consideraría de elección?
a) Extirpación total de la lesión.
b) Láser.
c) Crioterapia.
d) Conducta expectante.
e) Todas las opciones son correctas. 
De acuerdo con nuestros hallazgos, la aplicación estricta del acrónimo clínico ABCD no sería de utilidad en el reconocimiento del MDP. El uso rutinario de la dermatoscopia en la práctica diaria y su aplicación incluso en lesiones clínicamente banales permiten la detección de

\section{BIBLIOGRAFÍA}

1. Kopf AW, Welkovich B, Frankel RE, Stoppelmann EJ, et ál. Thickness of malignant melanoma: global analysis of related factors. J Dermatol Surg Oncol 1987;13:345-390.

2. Argenziano G, Soyer HP, Chimenti S, Talamini R, et ál. Dermoscopy of pigmented skin lesions: results of a consensus meeting via the Internet. JAm Acad Dermatol 2003;48:679-693.

3. Salerni G, Terán T, Puig S, Malvehy J, et ál. Meta-analysis of digital dermoscopy follow-up of melanocytic skin lesions: a study on behalf of the international dermoscopy society. J Eur Acad Dermatol Venereol 2013;27:805-814.

4. Friedman RJ, Rigel DS, Kopf AW. Early detection of malignant melanoma: the role of physician examination and selfexamination of the skin. CA Cancer J Clin 1985;35:130-151.

5. Nachbar F, Stolz W, Merkle T, Cognetta AB, et ál. The ABCD rule of dermatoscopy. High prospective value in the diagnosis melanomas con bajo índice de sospecha que los pacientes podrían desconocer. En el contexto de los pacientes de alto riesgo, el seguimiento digital hace posible la detección de los melanomas incipientes que carecen no solo de criterios clínicos, sino también dermatoscópicos de malignidad.

of doubtful melanocytic skin lesions. J Am Acad Dermatol 1994;30:551-559.

6. Rosendahl C, Cameron A, McColl I, Wilkinson D. Dermatoscopy in routine practice-'chaos and clues'. Aust Fam Physician 2012;41:482-487.

7. Salerni G, Alonso C, Fernández-Bussy R. A series of small-diameter melanomas on the legs: dermoscopic clues for early recognition. Dermatol Pract Concept 2015;31;5:31-36.

8. Megaris A, Lallas A, BagoliniLP,Balais G, etál.Dermoscopy features of melanomas with a diameter up to $5 \mathrm{~mm}$ (micromelanomas): A retrospective study. J Am Acad Dermatol 2020;83:1160-1161.

9. Salerni G, Terán T, Alonso C, Fernández-Bussy R. The role of dermoscopy and digital dermoscopy follow-up in the clinical diagnosis of melanoma: clinical and dermoscopic features of 99 consecutive primary melanomas. Dermatol Pract Concept 2014;31;4:39-46.

\section{DERMATÓLOGOS JÓVENES}

\section{Elección múltiple: PLACA ERITEMATOVIOLÁCEA EN UN DEDO / Respuestas}

\section{Respuestas correctas: 1) c; 2) b; 3) c; 4) e}

Los angioqueratomas (AQ) son proliferaciones vasculares benignas adquiridas del plexo vascular superficial, asociadas a una hiperplasia epidérmica suprayacente.

Según la última actualización de la International Society for the Study of Vascular Anomalies (ISSVA) 2018, permanecen como anomalías vasculares no clasificadas.

Existen variantes localizadas o difusas. Las localizadas comprenden: $\mathrm{AQ}$ de Mibelli, $\mathrm{AQ}$ solitario, $\mathrm{AQ}$ circumscriptum naeviforme, $A Q$ de la lengua y $A Q$ de Fordyce. La difusa, AQ corporis diffusum, se caracteriza por múltiples angioqueratomas distribuidos en forma similar a un traje de baño, y está relacionada con los trastornos de almacenamiento lisosómico, entre los que se incluye la enfermedad de Fabry.

El AQ solitario es la variedad más frecuente de todos los AQ (70-83\%). Afecta tanto a los niños como a los adultos entre los 20 y los 40 años. Si bien el traumatismo crónico se describió como un desencadenante, no siempre se encuentra esta asociación.
Se presenta como una pápula brillante, suave, no queratósica, que posteriormente crece y se torna queratósica, verrugosa, oscura y firme, de 2 a 10 mm de diámetro.

Se localiza con mayor frecuencia en las extremidades inferiores, aunque también puede ubicarse en el pene, el clítoris, la vulva y la cavidad bucal. Se han informado casos con afectación de las palmas y las plantas.

La presencia de una pápula o nódulo oscuro obliga al diagnóstico diferencial con otros tumores cutáneos, como los nevos melanocíticos, el nevo de Spitz-Reed, el melanoma, el carcinoma basocelular pigmentado, las queratosis seborreicas 0 el dermatofibroma, así como con otras lesiones vasculares, como el angioma de Cherry y el granuloma piógeno.

La dermatoscopia cumple una función importante para diferenciarlo de estas afecciones. Los hallazgos incluyen lagunas redondeadas bien delimitadas de color rojo oscuro (que se corresponden con vasos dérmicos dilatados sin trombosis), velo blanquecino (acantosis e hiperquera- tosis) y eritema (inflamación y extravasación de eritrocitos asociadas al trauma). La combinación de estos datos conforma tres posibles patrones dermatoscópicos: 1) lagunas oscuras y velo blanquecino; 2) lagunas oscuras, velo blanquecino y eritema periférico; y 3) lagunas oscuras, velo blanquecino y costras hemorrágicas.

El diagnóstico definitivo se realiza con el estudio histopatológico. Los hallazgos incluyen diferentes grados de hiperqueratosis o epidermis ortoqueratósica compacta, acantosis y la presencia de capilares dilatados congestivos de paredes delgadas en la dermis papilar. El AQ solitario no tiene asociaciones sistémicas ni se relaciona con deficiencias enzimáticas. Suele ser asintomático y benigno.

Aunque las complicaciones son poco frecuentes $y$ menores (sangrado, prurito y dolor), su apariencia puede generar molestias estéticas 0 ansiedad. No hay un tratamiento de primera línea. Pueden utilizarse métodos localmente destructivos: crioterapia, electrocoagulación, tratamiento con láser y escisión quirúrgica.

\section{BIBLIOGRAFÍA}

- Cuestas D, et ál. Angiokeratomas, not everything is Fabry disease. Int J Dermatol 2019;58:713-721.

- Piccolo V, et ál. Dermatoscopy of vascular lesions. Dermatol Clin 2018;36:389-395.

- Wang L, Gao T, Wang G. Solitary angiokeratoma on palms and soles: A clinicopathological analysis of 21 cases. J Dermatol 2013;40:653-656. 